\title{
Retraction Note: Research on tool change time and the dynamic reliability of the machining process based on sensitivity analysis
}

\author{
Xingang Wang ${ }^{1} \cdot$ Baoyan Wang ${ }^{1} \cdot$ Chunmei LV $^{1} \cdot$ Xiaoming Chen ${ }^{2} \cdot$ Yimin Zhang $^{1}$ \\ Published online: 25 June 2021 \\ (C) Springer-Verlag London Ltd., part of Springer Nature 2021
}

Retraction Note: Int J Adv Manuf Technol (2017) 89:1535-1544

https://doi.org/10.1007/s00170-016-9177-0

The Editor in Chief has retracted this article at the request of the corresponding author, Baoyan Wang. The authors found substantial errors which affect the results and conclusions of the study. Specifically, there has been an error in the results presented in Table 3 and 4 . This affects figs. 4-13 which are now incorrect. Further more, the values presented for 'manufacturing process reliability' have been miscalculated.

Baoyan Wang, Xingang Wang, Xiaoming Chen and Yimin Zhang agree to this retraction. Chunmei Lv did nor respond to any correspondence from the editor or publisher about this retraction.

Publisher's note Springer Nature remains neutral with regard to jurisdictional claims in published maps and institutional affiliations.

The online version of the original article can be found at https://doi.org/ $10.1007 / \mathrm{s} 00170-016-9177-0$

Baoyan Wang

huixiao_chen@126.com

1 Center of Mechanical Reliability \& Dynamics, Northeastern

University, Qinhuangdao 066004, China

2 School of Mechatronics Engineering, Harbin Institute of Technology, Harbin 150001, Heilongjiang, China 